epidemiologic and laboratory follow-up were possible, transmission from a $\mathrm{HCW}$ to a patient could not be documented. These data support earlier assessments that the risk of HIV transmission from an infected HCW to a patient is very small.

FROM: Robert L, et al. Investigations of patients treated by HIV-infected health care workers: an update. Abstracts of the First National Conference on Human Retroviruses and Related Infections. December 12-16, 1993; Washington, DC. Abstract 535.

\section{Transmission of TB from Flight Attendant to Crew}

According to an abstract submitted to the 33rd Interscience Conference on Antimicrobial Agents and Chemotherapy (ICAAC) held October 17-20, 1993, in New Orleans, a flight attendant with cavitary pulmonary tuberculosis may have transmitted TB to as many as 13 of 265 crew members on international flights. The investigation of transmission among coworkers was prompted by TB skin test (TST) conversions among six of seven household contacts of the flight attendant. The risk of infection for coworkers appeared to be greater for those exposed closer to the date of diagnosis of the flight attendant and with increasing hours of exposure. The authors report that studies to evaluate the risk of transmission to passengers are ongoing.

FROM: Driver C, et al. Airborne transmission of airborne disease-cavitary TB in a flight attendant. Abstracts of the 33rd Interscience Conference on Antimicrobial Agents and Chemotherapy (ICAAC). October 17-20, 1993; New Orleans, LA. Abstract 1369.

\section{Urine Tests Can Reveal Chlamydial Infection in Asymptomatic Men}

Dr. Mary Ann Shafer et al from the University of California, San Francisco, reported the results of a study recently published in the Journal of the A merican $M$ edical A ssociation that showed a combination of urine-based tests that are both clinically effective and cost-effective for detecting Chlamydia trachomatis in asymptomatic young men. As a simple alternative to the urethral swab method, performance of a dipstick test for leukocyte esterase (LE) or a microscopic exam showing polymorphonucleocytes on the first portion of a voided urine specimen predicts chlamydial infection in asymptomatic young men. The authors state that urethral tissue cultures cost $\$ 697$.

In the same journal, Dr. M. Genc et al reported a detailed economic evaluation that demonstrated that
LE-enzyme immunoassay screening of urine from asymptomatic young men followed by treatment with a single dose of azithromycin is a cost-effective strategy and leads to reduced overall health costs when the prevalence exceeds $2 \%$.

Based on these two published reports, Drs. Mark Aronson and Russell Phillips, in an accompanying editorial, recommend routine performance of a dipstick for LE in asymptomatic, sexually active, adolescent males and young men during all routine examinations.

FROM: Shafer MA. Evaluation of urine-based screening strategies to detect Chlamydia trachomatis among sexually active asymptomatic young males. JAMA 1993;270:2065; Genc M, et al. An economic evaluation of screening for Chlamydia trachomatis in adolescent males. JAMA 1993;270:2065; Aronson MD, Phillips RS. Screening young men for chlamydial infection. JAMA 1993;270:2097. Editorial.

\section{Outbreak of Legionellosis Associated with Exposure to Hot Tub}

Six undergraduate students experienced febrile illnesses while vacationing at a Vermont ski resort. Legionellosis was confirmed in all six students by serologic testing. One student with insulin-dependent diabetes mellitus had a clinical course consistent with Legionnaires disease and was ill for three weeks with severe pneumonia and hypoxemia. The other five students had self-limiting febrile illness without pneumonia, consistent with symptoms associated with Pontiac fever, and recovered within three to six days. The source of the Legionella pneumophila in this outbreak was believed to be a private hot tub.

While $L$ pneumophila is associated with pointsource outbreaks of either Pontiac fever or Legionnaires disease, these two forms of infection generally are considered to be clinically and epidemiologically distinct. The basis for the marked difference in the expression of disease is unknown. However, differences in bacterial strains, inoculum size, and host response have been proposed. The differing clinical presentation of one student compared with the other five in this outbreak likely was influenced by the immune status of the host.

$\mathrm{L}$ pneumophila recovered from whirlpool-type spas has been associated with other outbreaks of Pontiac fever and Legionnaires disease.

FROM: Thomas DL, et al. Hot tub legionellosis. A rch Intern M ed 1993;153:2597-2599. 